\title{
Propagation Effects in the Application of Weather Radar - Positive and Negative Impact
}

\author{
Martin Hagen ${ }^{1}$, Jens Reimann ${ }^{2}$ \\ ${ }^{1}$ DLR Institut für Physik der Atmosphäre, Oberpfaffenhofen, Germany, martin.hagen@dlr.de \\ ${ }^{2}$ DLR Institut für Hochfrequenztechnik und Radarsysteme, Oberpfaffenhofen, Germany
}

\begin{abstract}
Propagation effects play in important role in the application of weather radar. Attenuation and depolarization have negative effects on the quality of radar data and hinder rainfall estimation, whereas the differential propagation phase can be used for the quantification of precipitation and even correction of attenuation effects.
\end{abstract}

Index Terms - weather radar, propagation, measurement.

\section{INTRODUCTION}

Weather radar is used in Meteorology for the identification and quantification of precipitation systems like thunderstorms or weather fronts. Weather radars operate with centimeter wavelength. In order to observe non-precipitating clouds millimeter-wave radars are used.

The propagation of electromagnetic waves through the atmosphere and clouds or precipitation is affected by the respective media. While the primary objective of weather radar is the measurement of properties of precipitation like rain rate of hydrometeor type, propagation effects often hinder such measurements. For example, especially at short wavelength there is considerable attenuation in rainy situations. On the other hand, propagation effects are used to derive properties of clouds and precipitation.

In this paper we discuss common effects of propagation like attenuation, depolarization, and the usage of differential phase measurements for rain estimation or auto-calibration.

\section{WEATHER RADAR}

Weather radars are pulsed radars operating at centimeter waves, typical characteristics are shown in Tab. I and Tab. II. In contrast to aviation surveillance radar systems a volume filled with targets is observed and besides localization of the target a quantification of the target is aimed. More about weather radar can be found in [1], [2], and [3]. Advanced applications are outlined in [4].

Typically modern dual-polarization Doppler weather radar generate in near real-time a number of products which

TABLE I. FREQUENCY BANDS USED FOR WEATHER RADAR IN EUROPE

\begin{tabular}{|l|c|c|}
\hline Band & Frequency $\boldsymbol{f}(\mathbf{G H z})$ & Wavelength $\boldsymbol{\lambda}(\mathbf{c m})$ \\
\hline $\mathrm{X}$ & $9.3-9.5$ & $3.1-3.2$ \\
\hline $\mathrm{C}$ & $5.6-5.65$ & $5.3-5.4$ \\
\hline $\mathrm{S}$ & $2.7-2.9$ & $1.0-1.1$ \\
\hline
\end{tabular}

TABLE II. CHARACTERISTICS OF WEATHER RADAR

\begin{tabular}{|l|c|}
\hline Parameter & Value \\
\hline pulse peak power & $10-1000 \mathrm{~kW}$ \\
\hline pulse duration & $0.6-2 \mu \mathrm{s}$ \\
\hline pulse repetition frequency & $300-2500 \mathrm{~Hz}$ \\
\hline half-power beam width & $1-3^{\circ}$ \\
\hline maximum range & $100-300 \mathrm{~km}$ \\
\hline range resolution & $90-300 \mathrm{~m}$ \\
\hline antenna rotation speed & $2-6 \mathrm{rpm}$ \\
\hline
\end{tabular}

are normally displayed either in a geo-referenced PPI (plan position indicator), or interpolated to a constant altitude CAPPI (constant altitude plan position indicator). Often the reflectivity or rain rate product is composed to national or international radar composites.

\section{A. Reflectivity}

Radar reflectivity factor or shortly called reflectivity is the standard product for weather radars [1][2]. Reflectivity factor $z$ is defined as

$$
z=\sum_{V o l} D_{i}^{6}
$$

where $D$ is the diameter of the scattering particles and the sum is over a unit volume of $1 \mathrm{~m}^{3}$. Thus the unit is $\mathrm{mm}^{6} / \mathrm{m}^{3}$. Commonly the logarithmic presentation in $\mathrm{dB}$ is used relative to $1 \mathrm{~mm}^{6} / \mathrm{m}^{3}$. The respective unit is then $\mathrm{dBz}$. Reflectivity is used to locate precipitation systems.

\section{B. Rain Rate}

For meteorological usage reflectivity factor $\mathrm{z}$ is converted to rain rate $R$. Rain rate is defined as the flux of liquid water through a unit plane and normally expressed in $\mathrm{mm} / \mathrm{h}$ or liter per hour per square meter. Since $z$ and $R$ are different moments of the a priori unknown rain drop size distribution, a linear conversion from $z$ to $R$ is not possible. Normally empirical $z-R$ relations in the form of

$$
z=a R^{b}
$$


are used [1]. $a$ is in the order of 100 to 300 , and $b$ in the order of 1.2 to 1.6 , depending on the kind of precipitation, the climatology and the location [1]. Due to the non-linear relation, the uncertainty in rain rate estimation is in the order of $10-50 \%$.

\section{Differential Reflectivity}

Weather radar use linear polarized waves. Modern weather radar use linear horizontal and vertical polarized waves, either simultaneous transmitted and received (STAR mode), or alternating from pulse to pulse (AHV mode). Differential reflectivity $Z_{D R}$ is defined as the logarithmic ratio between the reflectivity at linear horizontal polarization $z_{H}$ and reflectivity at linear vertical polarization $z_{V}$

$$
Z_{D R}=10 \log _{10}\left(z_{H} / z_{V}\right)
$$

Since raindrops larger than $1 \mathrm{~mm}$ in diameter have an oblate shape, $Z_{D R}$ will be positive in rain. Differential reflectivity is used to improve rain rate estimation [5] and for the identification of hydrometeor type.

\section{Linear Depolarization Ratio}

In case of elongated scattering particles which are not aligned with the polarization plane, i.e. the minor axis elements $S_{H V}$ and $S_{V H}$ of the scattering matrix are non-zero, depolarization of the linear polarized wave takes place and the wave becomes elliptical and contains also a cross-polar component. The linear depolarization ratio $L D R$ is defined as

$$
L D R=10 \log _{10}\left(z_{V H} / z_{H H}\right)
$$

with $z_{H H}$ the co-polar and $z_{V H}$ the cross-polar reflectivity. In precipitation, $L D R$ is strong when ice particles are melting and start tumbling during fall. $L D R$ is a good indicator for hail shafts and the melting layer (bright band).

\section{E. Doppler Velocity}

If there is a relative motion between the radar and the target, the frequency of the received wave is changed by the Doppler Effect. Weather radar typically measures the phase difference between two consecutive pulses and estimate Doppler frequency which is then related to the radial motion of the precipitation particles. In general it is assumed that precipitation particles follow with the wind and have a known terminal fall velocity. Thus it is possible to estimate the wind field using measurements of Doppler velocity. Phase measurements, i.e. Doppler velocity are basically immune to propagation effects.

\section{F. Differential propagation phase}

When electromagnetic waves pass through precipitation particles, their propagation speed is delayed according the index of refraction. Depending on the shape and mass of the particle the propagation speed of the horizontal and vertical polarized wave will be different e.g. in case of oblate rain drops [6]. Differential propagation $\phi_{D P}$ phase is defined as

$$
\phi_{D P}=\phi_{H}-\phi_{V}
$$

The specific differential propagation $K_{D P}$ phase is the range derivative of $\phi_{D P}$

$$
K_{D P}=\frac{\phi_{D P}\left(r_{2}\right)-\phi_{D P}\left(r_{1}\right)}{2\left(r_{2}-r_{1}\right)}
$$

with $r$ as the range to the target volume. The unit is $\% / \mathrm{km}$. It is assumed that the scattering process itself does not introduce a differential phase shift. This is true as long the scattering particles are small compared to the wave length. Differential phase is used for attenuation correction and rain rate estimation.

\section{PROPAGATION EFFECTS}

Propagation effects play an important role in the application of weather radar. Commonly known is the attenuation of the signal during the passage of rain cells. This negative effect on reflectivity measurements can be corrected to some degree using reflectivity. On the other hand the differential propagation phase is a well suited parameter to correct for attenuation or to estimate rain rate and $L D R$ helps the identification of hydrometeors.

\section{A. Negative Propagation Effects}

Negative propagation effects are those effects hindering or contaminating weather radar measurements.

\section{1) Attenuation}

Attenuation is the reduction of the transmitted energy during the passage through a media. The forward transmitted energy is reduced by the back and sideward scattered energy and the absorption of energy by the scattering particles. No attenuation will occur in vacuum.

Here we will concentrate on the attenuation in rain since the scattering processes of rain drops can be described quite easily. Absorption is proportional to $D^{3}$, whereas reflectivity is $\propto D^{6}$. Consequently, absorption cannot be estimated directly form reflectivity measurements. A number of empirical relations have been proposed for weather radar.

The following example (Fig. 1) shows the effect of

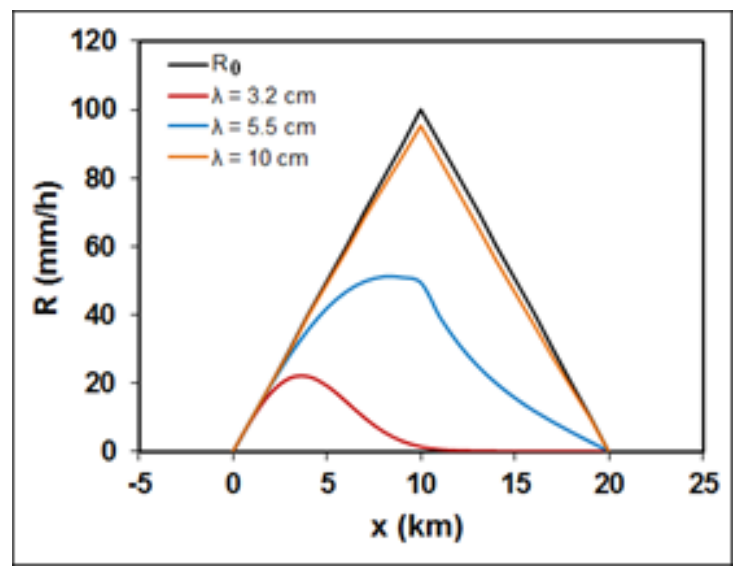

Fig. 1. Simulated effect of attenuation on rain rate measurements by weather radar. 
attenuation on the estimation of the rain rate using a $z-R$ relation. A simulated thunderstorm cell with a maximum rain rate of $100 \mathrm{~m} / \mathrm{h}$ at the center and a width of $20 \mathrm{~km}$ cannot be resolved with $\mathrm{C}$ and $\mathrm{X}$ band weather radars. Even the position of the core is incorrectly located with a X-band radar. Almost no attenuation takes place for S-band radars. The maximum precipitation is considerable underestimated with $\mathrm{X}$ and C-band radars. Short-term forecasting for hydrological purpose might fail when using radars with short wavelengths $\lambda$.

Additionally, differential attenuation is observed in measurements of $Z_{D R}$ when the wave passes through heavy precipitation with large oblate raindrops. In this situation the horizontal wave is much more attenuated than the vertical polarized wave. Thus resulting in a weaker horizontal signal at the end of the precipitation core and leading to negative $Z_{D R}$ in weak precipitation beyond the cell. Note, that particles with negative $Z_{D R}$ do not occur in nature.

Due to the non-linear relation between reflectivity and attenuation, a direct correction for attenuation is not possible. The application of simple empirical relations can lead to an unstable behavior introducing large errors [7]. Certain filtering of data is necessary before attenuation correction can be applied.

\section{2) Depolarization}

As shown above, $L D R$ is a measure for depolarization of the linear transmitted wave. However, it was found that also $Z_{D R}$ is affected by depolarization in case the radar operates in the STAR mode. The basic assumption for measurements in STAR mode is that the linear horizontal and linear vertical polarized waves (with same frequency) propagate without any interaction. This is not true anymore in case of depolarization where cross-talk between the two polarizations take place. A further assumption is that the waves are transmitted with equal phase, i.e. the resulting wave is a linear polarized wave with $45^{\circ}$ orientation. In case a $90^{\circ}$ phase shift is between the horizontal and vertical wave, the resulting wave is circular polarized. In the past radar manufacturers did not respect the phase difference between the two channels and their cross-coupling. However, the coupling of the cross-polar terms with the co-polar terms depends on the phase between the two channels.

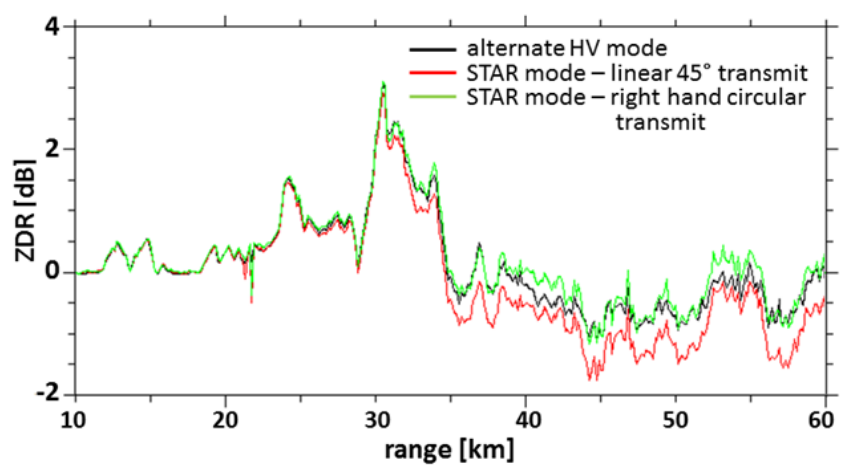

Fig. 2. Profile of $Z_{D R}$ measurements with POLDIRAD on 2012-06-04 at 15:46 using three diferent polarization modes. For details see text.
With the DLR research radar POLDIRAD [8] it is possible to measure the STAR mode with different phase offsets simultaneous with the AHV mode [9]. In AHV mode the complete scattering matrix can be measured, whereas in STAR mode only the major axis elements possible contaminated by the minor axis elements can be measured.

Fig. 2 shows a single ray through a convective cell embedded in stratiform precipitation. $Z_{D R}$ was measured in three different ways: a) using the STAR mode with an initial phase offset of $0^{\circ}$, i.e. linear $45^{\circ}$ orientation; b) using the STAR mode with an initial phase offset of $90^{\circ}$, i.e. circular polarization; c) using the AHV mode avoiding cross-channel contamination. While the offset-corrected profile of $Z_{D R}$ of all three methods overlap prior to the convective cell at range $30 \mathrm{~km}$, distinct offset in the order of up to $1 \mathrm{~dB}$ can be observed on the rear side of the cell. This is caused by the change of the phase between the two measurements in STAR mode. AHV mode is immune to phase changes along the propagation phase. Note that rain rate estimation proposed by [5] requires high quality $Z_{D R}$ measurements within $0.1 \mathrm{~dB}$ accuracy.

\section{B. Positive Propagation Effects}

Positive effects are considered as those effects which help to measure properties of precipitation.

\section{1) Rain rate estimation using $K_{D P}$}

As shown by [6] and others, $K_{D P}$ can be used to estimate rain rate with higher precision than it is possible with $Z$ alone. The reason is that $K_{D P}$ is nearly proportional to the mass of the raindrops, thus less sensitive on the raindrop size distribution. For C-band an empirical relation

$$
R=19.8 K_{D P}
$$

was found by [10]. The linear relation shows the independence of the raindrop size distribution. However, the coefficient of 19.8 shows that $K_{D P}$ has to be estimated with high precision in order to retrieve low rain rates. Common practice is to smooth measured $\phi_{D P}$ over a range of a few kilometers to improve accuracy of $K_{D P}$. Since $K_{D P}$ is immune to attenuation effects it is well suited even for high precipitation rates.

\section{2) Attenuation correction using $K_{D P}$}

Attenuation is proportional to the mass of precipitation $\left(\propto D^{3}\right)$ and $K_{D P}$ is roughly proportional to $D^{4}$. Therefore, a nearly linear relation between $K_{D P}$ and attenuation of reflectivity $\alpha_{H}$ and differential attenuation $\alpha_{D}$ of $Z_{D R}$ can be found [11]. Using attenuation correction to $Z$ and $Z_{D R}$, both parameters can be used further for rain rate estimation with high spatial resolution or for the identification of hydrometeors.

Fig. 3 shows one example of a radar ray through a strong convective cell. Using $\alpha_{H}=0.12$ and $\alpha_{D}=0.04$ a corrected profile of $Z$ and $Z_{D R}$ can be estimated. With this correction a more realistic positive $Z_{D R}$ is obtained at the rear side of the convective cell. $Z$ is increased by more than $10 \mathrm{~dB}$ within the 


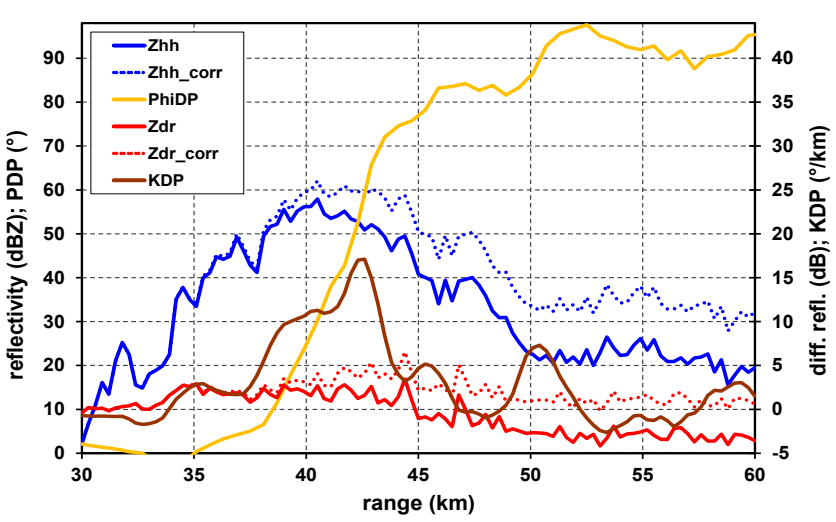

Fig. 3. Range profile measured with POLDIRAD on 2007-08-15 at 18:21.

convective cell; this certainly has a considerable impact for hydrological applications: $40 \mathrm{dBz}$ at range $45 \mathrm{~km}$ corresponds roughly to $13 \mathrm{~mm} / \mathrm{h}$ rainfall, whereas $50 \mathrm{dBz}$ corresponds to about $67 \mathrm{~mm} / \mathrm{h}$ rainfall. With this heavy rainfall, flooding in the vicinity of small rivers can occur; uncorrected radar data would not raise a warning level in this situation.

\section{3) Self-consistency check using $Z, Z_{D R}$, and $\phi_{D P}$}

Self-consistency or auto-calibration of radar parameters is a further application of differential phases. It was proposed by [11] and [12] to use the observed values of $Z$ and $Z_{D R}$ to predict $K_{D P}$ at each range bin. The estimated $K_{D P}$ values are then used to construct the total differential phase $\phi_{D P}$ along the radar beam. Comparing the measured profile of $\phi_{D P}$ with the reconstructed profile of $\phi_{D P}$ allows identifying misscalibration of reflectivity and $Z$ can be adjusted accordingly. It has to be assured that $Z_{D R}$ is properly calibrated, this is normally checked with a vertically pointing antenna in light rain where $Z_{D R}$ should be zero.

\section{SUMMARY AND CONCLUSIONS}

In weather radar applications, propagation effects are often considered only in case of attenuation for X-band radars. However, this is only one aspect. Attenuation occurs at all wavelengths, even though it is of minor importance for S-band radars. The application of attenuation correction procedures using differential phase measurements allow for a successful correction of attenuation at least for rain. Other hydrometeors like hail or graupel can have a large variety of shapes and will have also different scattering properties in case they are coated with a thin water layer. Depolarization of linear waves propagating through non-spherical hydrometeors, or hydrometeors which are not within the Rayleigh regime $(D<<\lambda)$ has an impact on the accuracy of $Z_{D R}$ measurements with weather radar systems operating in STAR mode.

In summary, propagation effects do contaminate power measurements like $Z$ and $Z_{D R}$, but on the on the other hand, modern dual-polarization Doppler weather radar systems benefit from applications using the differential propagation phase.

\section{REFERENCES}

[1] Battan, L. J. 1973: Radar observation of the atmosphere. University of Chicago Press, Chicago, USA

[2] Doviak, R. J., and D. S. Zrnic, 1993: Doppler Radar and Weather Observations, 2nd ed., Academic, San Diego, USA.

[3] Bringi, V. N. and Chandrasekar, V., 2001: Polarimetric Doppler Weather Radar - Principles and Applications. Cambridge University Press, 636 pp.

[4] Meischner, P. (ed.), 2004: Weather radar: Principles and advanced applications. Springer, Berlin, 337 pp.

[5] Seliga, T. A. and V. N. Bringi, 1976: Potential use of radar differential reflectivity measurements at orthgonal polarizations for measureing precipitation. J. Appl. Meteor., 15, 69.

[6] Sachidananda, M., and D.S. Zrnic, 1986: Differentail propagation phase shift and rainfall rate estimation. Radio Sci., 21, 235-247.

[7] Hildebrand, P. H., 1978: Iterative correction for attenuation of $5 \mathrm{~cm}$ radar in rain. J. Appl. Meteor., 17, 508-514.

[8] Schroth, A. C., M. Chandra, and P. F. Meischner, 1988: A c-band coherent polarimetric radar for propagation and cloud physics research. J. Atmos. Oceanic Technol., 5, 803-822.

[9] Reimann, J., 2013: On fast, polarimetric non-reciprocal calibration and multipolarization measurements on weather radars. Ph.D. dissertation, Technische Universität Chemnitz, $161 \mathrm{pp}$.

[10] Scarchilli, G., E. Gorgucci, V. Chandrasekar, and T.A. Seliga, 1993 Rainfall estimation using polarimetric techniques at C-band frequencies. J. Appl. Meteor., 32, 1150-1160.

[11] Gorgucci, E., Scarchilli, G., Chandrasekar, V., Meischner, P., Hagen, M., 1998: Intercomparison of techniques to correct for attenuation of C-band weather radar signals. J. Appl. Meteor., 37, 845-853.

[12] Goddard, J.W.F., J. Tan and M. Thurai, 1994: Technique for calibration of meteorological radars using differential phase. Electron. Lett., 30, 166-167. 